\title{
Nasal Lobular Capillary Hemangioma as a Complication after an Endoscopic Transsphenoidal Gonadotrophin-Producing Pituitary Adenoma Resection
}

\author{
Dalila Forte ${ }^{1}$ Amets Sagarribay Irañeta ${ }^{1}$ Anabela Nabais ${ }^{1}$ António Figueiredo ${ }^{2}$ Manuela Mafra ${ }^{2}$ \\ Victor Gonçalves ${ }^{1}$ \\ ${ }^{1}$ Department of Neurosurgery, Centro Hospitalar de Lisboa Central, \\ Lisboa, Portugal \\ 2 Department of Pathology, Centro Hospitalar de Lisboa Central, \\ Lisboa, Portugal \\ Address for correspondence Dalila Forte, MD, Department of \\ Neurosurgery, Centro Hospitalar de Lisboa Central, R. José António \\ Serrano, Lisboa 1150-199, Portugal (e-mail: dalila.forte@gmail.com). \\ J Neurol Surg Rep 2017;78:e52-e54.
}

\begin{abstract}
Background Lobular capillary hemangioma is a rare benign tumor, most frequently located in the head or neck region, the nasal cavity being uncommonly affected. Its etiopathogenesis is not fully established, although traumatic and hormonal factors have been implied.

Case Description A 50-year-old female patient underwent an uneventful endoscopic transsphenoidal removal of a pituitary cystic macroadenoma at our institution. Nasal packing was used in postoperative hemostasis. Histopathology was compatible with a gonadotro-

\section{Keywords}

- lobular capillary hemangioma

- pyogenic granuloma

- endoscopic transsphenoidal pituitary surgery phin-producing adenoma. One month after the surgery, the patient presented with frequent episodes of epistaxis and a progressively growing nasal mass, which was removed endoscopically. Its pathological examination confirmed a lobular capillary hemangioma. Conclusions The authors present a clinical case combining two possible predisposing factors to the development of a nasal lobular capillary hemangioma: local traumatic injury through surgery and postoperative nasal packing and hormonal influence. This lesion is a rare complication of endoscopic transsphenoidal resections of pituitary adenomas.
\end{abstract}

\section{Introduction}

Lobular capillary hemangioma (LCH), also known as pyogenic granuloma, is a benign, rapidly growing, hypervascular lesion. Its usual locations are the skin and mucous membranes of the head and neck, the nasal cavity being a rare site of origin. ${ }^{1,2}$ Its etiopathogenesis is not fully established, although an association with traumatic and hormonal factors has been identified. These lesions tend to occur more frequently after nasal surgery or nasal packing and in women during pregnancy or taking oral

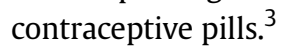

Here, we present the first reported case of a nasal LCH following an endoscopic transsphenoidal resection of a gonadotrophin-producing pituitary adenoma, reflecting a possible but rare complication of this procedure.

\section{Case Report}

A 50-year-old female patient presented with progressive loss of visual acuity. The neurologic examination revealed a bitemporal hemianopsia and the laboratory studies, including endocrinological investigation were normal. She was diagnosed with a pituitary cystic macroadenoma and underwent an endoscopic transsphenoidal removal of the lesion in our institution by a team of two neurosurgeons experienced in endoscopic procedures. The surgery and immediate received

November 1, 2016

accepted after revision

February 2, 2017
DOI http://dx.doi.org/

10.1055/s-0037-1600913.

ISSN 2193-6358. (c) 2017 Georg Thieme Verlag KG Stuttgart · New York
License terms

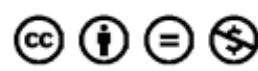




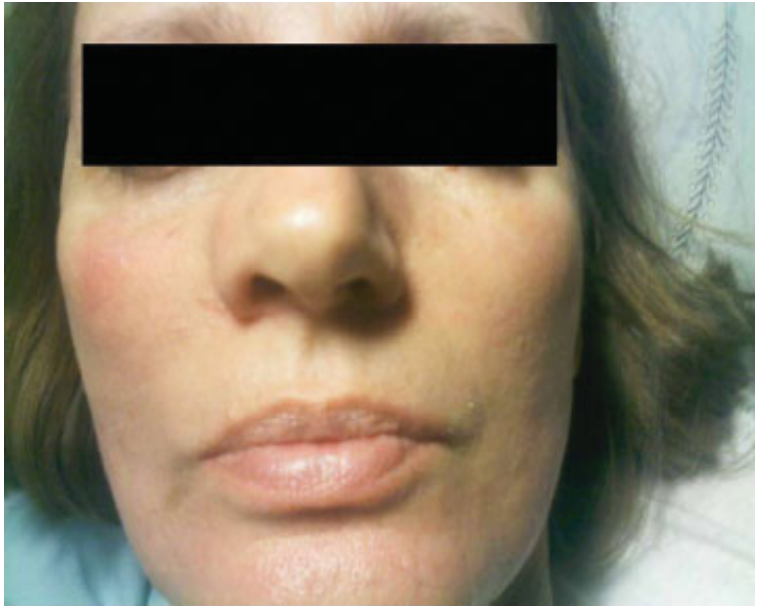

Fig. 1 Picture showing the right nasal mass.

postoperative recovery occurred without complications. Nasal packing was done bilaterally with Merocel (Medtronic) (polyvinyl-alcohol sponge) nasal tampon lubricated with antiseptic cream, maintained for 5 days and then removed uneventfully. The histopathologic review established the diagnosis of a gonadotropin (luteinizing hormone and follicle-stimulating hormone)-producing adenoma.

One month after the surgery, the patient presented with a progressively growing right nasal mass (-Fig. 1) and frequent intermittent episodes of nasal bleeding. Physical examination revealed a reddish, smooth-surfaced mass, filling the right nasal cavity. The mass was mobile and appeared pedunculated with its base attached to the anterior portion of the nasal septum. A nasal endoscopy was performed and a hemorrhagic right septal polyp was identified. The polyp was removed surgically, under general anesthesia and using nasal forceps and electrical cautery. The technique included partial resection of mucous septum and perichondrium. Pathological examination confirmed a LCH (-Fig. 2). Nasal packing was maintained for 4 days and then removed. She presented again with recurrent abundant epistaxis. Her blood pressure was normal, hemoglobin levels were discretely low, and coagulation tests were normal. The bleeding was controlled with the insertion of a nasal inflatable balloon catheter and cautery with silver nitrate. Nasal endoscopy was repeated after 1 week, 6 months, and 1 year, showing no signs of lesion recurrence. The patient remains asymptomatic at 1-year follow-up.

\section{Discussion}

LCH is a benign, hypervascular lesion. Grossly, it appears as a friable polypoid mass, which can present ulcerations on its surface. On histopathological examination its typical pattern is characterized by multiple, varying-sized capillaries in a lobular arrangement, surrounded by a loose fibromyxoid stroma. ${ }^{4}$ The most common locations for these lesions are the skin and mucous membranes of head and neck, particularly the gingiva of the oral cavity, the nasal cavity being a

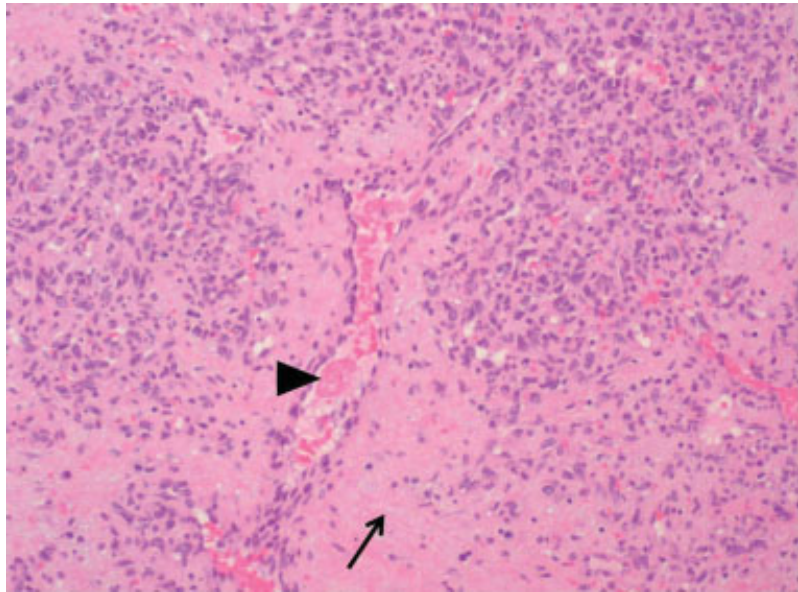

Fig. 2 Hematoxylin-eosin staining revealing the typical pattern of LCH-lobules of capillaries (arrowhead) surrounded by loose fibrous stroma (arrow). LCH, lobular capillary hemangioma.

rare site of origin. ${ }^{1,2}$ Within the nasal cavity, these lesions have been described to arise more frequently from the anterior portion of the nasal septum (Little area) or the anterior portion of the inferior turbinate. ${ }^{5,6}$ It can occur at all ages and affects both the sexes equally. ${ }^{2}$

It presents usually by unilateral recurrent epistaxis and nasal obstruction, though in some cases smell alterations can be involved. ${ }^{1}$ The lesion is usually identified on direct examination of the anterior nasal cavity. ${ }^{7}$

The exact etiopathogenesis of this lesion is unclear, although some predisposing factors have been implied, such as local trauma or hormonal influences. Regarding traumatic factors, the most commonly reported are nose picking and nasal packing, which are thought to trigger an inflammatory process in response to traumatic injury to the surface exposed. ${ }^{2,7}$ Also, hormonal stimulation has also been implicated, with some cases reported in association to pregnancy and oral contraception. ${ }^{8}$ In this case, the lesion developed after an endoscopic transsphenoidal pituitary access, which induces traumatic injury to the nasal mucosa both through intraoperative manipulation and postoperative nasal packing. The pituitary adenoma removed was gonadotrophin-producing which can also provide a favorable hormonal environment.

The differential diagnosis of an exophytic hypervascular mass in the nasal cavity presenting with intermittent episodes of epistaxis includes juvenile nasopharyngeal angiofibroma, which occurs typically in adolescent male patients, angiomatous polyp and rare malignant lesions, such as nasopharyngeal carcinoma or metastasis. ${ }^{1,7}$

Imaging studies might be required in larger lesions which pose diagnostic difficulties and require an evaluation of extent and relation to adjacent structures. ${ }^{6}$ CT-scanning reveals an intensely enhancing mass and an iso- or hypoattenuating cap of variable thickness. Bony changes (erosion or displacement) are not uncommon. ${ }^{3}$

Treatment consists of total surgical excision that can be achieved through direct visualization endoscopically without significant complications. This technique might also be 
e54 Nasal LCH as a Complication after Pituitary Adenoma Resection Forte et al.

used in follow-up and early detection of recurrences, which are uncommon after complete excision. ${ }^{1,7}$ Pregnancy-related lesions might regress spontaneously after delivery. ${ }^{8}$

\section{Conclusions}

This clinical case combines two predisposing factors to the development of a nasal LCH-hormonal influence (gonadotrophin-producing pituitary adenoma) and local traumatic injury (surgery and postoperative nasal packing).

Nasal LCH is a rare but possible complication of endoscopic transsphenoidal resection of pituitary adenomas.

\section{References}

$1 \mathrm{Chi} \mathrm{TH}$, Yuan CH, Chien ST. Lobular capillary hemangioma of the nasal cavity: a retrospective study of 15 cases in Taiwan. Balkan Med J 2014;31(01):69-71
2 Rallis E, Balatsouras D, Homsioglou E, Karapantzos I, Danielidis V. Lobular capillary hemangioma of the nasal cavity. J Otolaryngol 2005;34(03):194-195

3 Lee DG, Lee SK, Chang HW, et al. CT features of lobular capillary hemangioma of the nasal cavity. AJNR Am J Neuroradiol 2010; 31(04):749-754

4 Nayak DR, Bhandarkar AM, Shivamurthy A, Joy J. Intranasal lobular capillary haemangioma. BMJ Case Rep 2014. Doi: 10.1136/bcr-2014-207196

5 Patil P, Singla S, Mane R, Jagdeesh KS. Nasal lobular capillary hemangioma. J Clin Imaging Sci 2013;3:40

6 Puxeddu R, Berlucchi M, Ledda GP, Parodo G, Farina D, Nicolai P. Lobular capillary hemangioma of the nasal cavity: A retrospective study on 40 patients. Am J Rhinol 2006;20(04): 480-484

7 Lee HM, Lee SH, Hwang SJ. A giant pyogenic granuloma in the nasal cavity caused by nasal packing. Eur Arch Otorhinolaryngol. 2002;259(05):231-233

8 Delbrouck C, Chamiec M, Hassid S, Ghanooni R. Lobular capillary haemangioma of the nasal cavity during pregnancy. J Laryngol Otol 2011;125(09):973-977 